\title{
Trajectory Phase-Plane Method - Based Analysis of Stability and Performance of a Fuzzy Logic Controller for an Anti-Lock Braking System
}

\author{
Andrei Aksjonov \\ Innovations \\ Škoda Auto a.s. \\ Mladá Boleslav, Czech Republic \\ andrei.aksjonov@skoda-auto.cz \\ Valery Vodovozov \\ Department of Electrical Power Engineering and Mechatronics \\ Tallinn University of Technology \\ Tallinn, Estonia \\ valery.vodovozov@taltech.ee
}

\author{
Vincenzo Ricciardi \\ Department of Automotive Engineering \\ Technische Universität Ilmenau \\ Ilmenau, Germany \\ vincenzo.ricciardi@tu-ilmenau.de \\ Klaus Augsburg \\ Department of Automotive Engineering \\ Technische Universität Ilmenau \\ Ilmenau, Germany \\ klaus.augsburg@tu-ilmenau.de
}

\begin{abstract}
The trajectory phase-plane method provides an increased significance to the analysis of stability and performance of the controllers for complex nonlinear plants. The aim of this study is the stability and performance analysis of a fuzzy logic controller for anti-lock braking system applying the trajectory phase-plane technique. The controller's main task is to hold optimal braking performance by recognizing road surface. The stability and performance of the anti-lock braking system controller are examined with respect to the conventional friction brake and electric motors separately. The applied methodology allows for visual assessment of the stability and performance of studied nonlinear systems. The work refers to hardware-in-theloop simulations conducted at the Technische Universität Ilmenau. The results demonstrate that the fuzzy logic controller is stable independently of the employed actuator. Moreover, the electric actuators allow for faster convergence and more accurate tracking of the optimal wheel slip.
\end{abstract}

Keywords-Fuzzy control, phase-plane, anti-lock braking system, intelligent control, electric vehicles, stability analysis, performance analysis.

\section{INTRODUCTION}

An anti-lock braking system (ABS) aims at maximizing vehicle's braking effectiveness with preserved steerability. An excessive braking torque applied to the wheels leads to wheels' lockage, which, in turn, deteriorates the vehicle lateral stability and negatively affects the braking distance. Therefore, the vehicle is neither capable of steering nor stopping as fast as requested by the driver.

The first ABS was eventually applied in 1978 [1] in the wake of consolidate experience gained from the aerospace industry. During recent years, a constant development of ABS components and actuators, along with advanced control techniques has arisen [2]. Nowadays, a novel ABS encompasses more sophisticate control systems based on computational intelligence algorithms (e.g. fuzzy set theory, artificial neural networks, etc.), new actuators exhibit faster dynamics and cleaner technologies such as the regenerative brakes, which is a distinctive feature of hybrid and fully electric vehicles (EVs).

Most of the studies available in the literature evaluate performance of an ABS control by observing the system response in the time domain for specific braking maneuvers [3] - [7]. During the recent years, given the increasingly faster dynamics of the new brake actuators, techniques based on the analysis of the wheel slip value occurrence or the frequency response, turned out to provide a meaningful description of the system performance [2], [8], [9]. The advantage of these methods is their ability to analyze a long-time frame by conveniently compact representation, neglecting the time coordinate.

In the present work, the authors set out to elaborate further the conclusions drawn in [8] by focusing on control stability problem of fuzzy logic control (FLC) for ABS. Moreover, the authors also point out, how different dynamics between conventional friction (FB) and regenerative braking (RB) impacts the control stability and performance? Traditional stability analysis methods, like Lyapunov's direct or indirect methods, were applied to FLC previously [10], [11]. However, the trajectory phase-plane (TPP) method was rarely addressed in the literature [8]. Therein, the authors assess the stability of the existing ABS controller [3] by representing the slip tracking error versus the tracking error derivative. In [8], the stability condition is stated as follows: if the control is capable to stabilize the error and its derivative around the origin, then the control is stable. On the other hand, controller's performance is declared as: the ability of the controller to prompt convergence to an equilibrium point.

In this paper, the TPP is applied to visually assess the stability properties and convergence performance. The studied method is particularly suitable for non-linear systems whereby analytical solutions might not exist. The ABS FLC is designed for a full electric sport utility vehicle (SUV) equipped with four 
on-board motors and electro-hydraulic brakes (EHB) [3]. The developed controllers allow for blended control of electric motors and friction brakes in compliance with the driver's deceleration request. The presented analysis refers to simulations conducted on the hardware-in-the-loop (HIL) platform at Technische Universität Ilmenau (Germany). The HIL consist of an EHB system interfaced with the vehicle dynamics simulator IPG CarMaker ${ }^{\circledR}$ (Karlsruhe, Germany).

The paper is structured as follows. The following Section describes vehicle model development and HIL environment. Section III delivers a brief introduction to an FLC for ABS (for more details on the adopted control method, the readers are encouraged to study [3]). The stability and performance analysis problems are stated in Section IV. Section V is dedicated to HIL experimental results. Finally, the paper is concluded in Section VI.

\section{VEHICLE MODEL AND HIL SETUP}

\section{A. Dynamics of a Braked Wheel}

A simplified drawing of a single-wheel model is presented in Fig. 1. In case of straight line braking, the torque balance about a wheel axis is [12]:

$$
J_{W} \dot{\omega}_{W}=r_{W} F_{X}-T_{B},
$$

where $J_{W}$ is moment of inertia of wheel; $\omega_{W}$ is angular velocity of wheel; $T_{B}$ is braking torque, $r_{W}$ is radius of deformed tire; $F_{X}$ is longitudinal force of tire.

For EV, $T_{B}$ is the sum of regenerative $T_{R B}$ and friction $T_{F B}$ braking torques. Modern vehicles are not equipped with appropriate sensors to measure $T_{R B}$ and $T_{F B}$ directly. However, these variables are proportional to the phase current of an electric drive and the brake's line pressure, respectively [13]. Both variables are measured with available sensors [14]. Here, the controller signals are directly expressed as $T_{R B}$ and $T_{F B}$.

\section{B. State Observation}

Efficient ABS control depends on tire-road friction coefficient $\mu$, which can be calculated as the ratio between

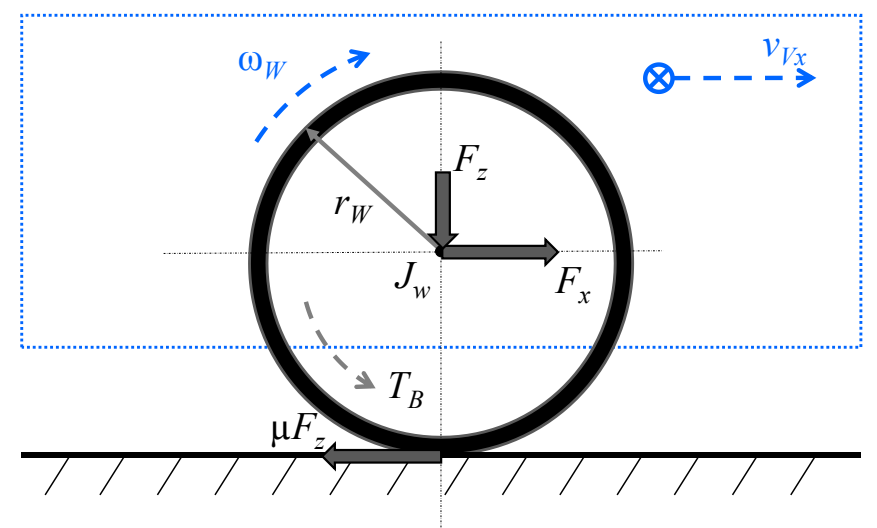

Fig. 1. A schematic drawing of a braked wheel (single-wheel model). longitudinal and vertical forces applied to the wheel [6]:

$$
\mu=\frac{F_{X}}{F_{Z}}=\frac{a_{V x}}{g},
$$

where $F_{X}$ is longitudinal tire force, $F_{Z}$ is normal tire force; $m_{V}$ and $a_{V x}$ are mass and longitudinal acceleration of vehicle, respectively; $g$ is gravitational acceleration.

The idea behind the proposed control method is to refer the road surface estimation to the maximum deceleration rate that the vehicle experiences during braking. Consequently, the maximum deceleration rate is used to estimate the road adhesion peak by an FLC. In this paper, the associated variable is defined as $\mu^{*}$.

In case of deceleration, the longitudinal wheel slip $\lambda$ is calculated as [13]:

$$
\lambda=\frac{v_{V x}-v_{W x}}{v_{V x}},
$$

where $v_{V x}$ is the longitudinal vehicle velocity found according to [14]:

$$
v_{V x}=\int a_{V x} d t
$$

and the longitudinal wheel speed $v_{W x}$ is derived as:

$$
v_{W x}=r_{W} \omega_{W} .
$$

\section{Vehicle Model}

The studied vehicle is a full electric SUV equipped with four independently controlled on-board switched reluctance motors (SRM). Its simplified scheme with the control links is

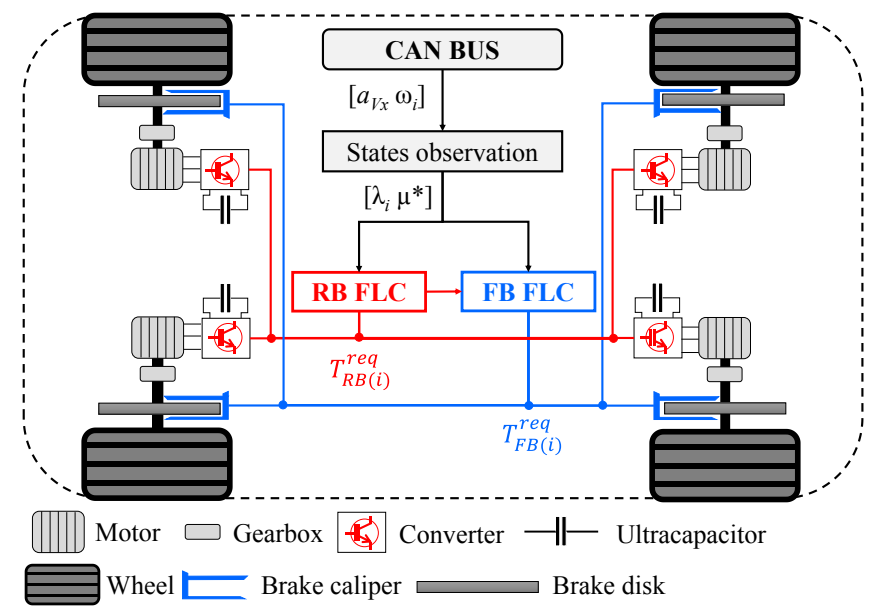

Fig. 2. Simplified scheme of the four on-board motors powertrain architecture with control links: CAN BUS - controller area network bus; RB FLC - regenerative braking fuzzy logic controller; FB FLC - friction braking fuzzy logic controller. 
depicted in Fig. 2. Each SRM is connected to the wheel hubs through a half-shaft transmission. The 14 degrees-of-freedom vehicle model is built in IPG CarMaker ${ }^{\circledR} 6.0$ (Karlsruhe, Germany), and is parametrized according to the EV manufacturer specifications, which can be found in [3].

The simulator employs an experimentally validated tire model based on Pacejka's Magic Formula 6.1 [15]. In Fig. 3, the slip-force curves on common road surfaces (i.e. dry, damp, wet, and icy) are reported. The optimal slips lay on the peaks of the curves as depicted in Fig. 3. According to the road surface adhesion coefficient, it is possible to identify a negative gradient region where the wheel dynamics exhibit an unstable behavior. It is worth mentioning that during braking, the applied torque might exceed the tire-road friction limit leading to a reduction of the wheel rotational speed until complete wheel lockage and deterioration of lateral stability. To avoid the undesired jerky behavior, the wheel slip ratio is constrained in the stable region throughout the vehicle braking process by means of a proper tuning of the adopted ABS FLC. Moreover, the closer the value of the wheel slip is to the optimal one, the more effective the braking is [3], [15].

The EV model includes four on-board motor models. The experimentally obtained SRM characteristics are described in details in [3]. Its behavior is featured through a first-order transfer function. The maximum achievable torque on the wheels is $2100 \mathrm{Nm}$. At last, to recreate realistic dynamics of the brake lining coefficient of friction, the Ostermeyer's model is improved with data collected from the brake dynamometric testbed available at Technische Universität Ilmenau (Germany). The model takes into consideration the brake linings' coefficient of friction dependence against speed, pressure and temperature. Thus, it allows for an improved loyalty of the HIL test bed [16].

\section{Experimental Setup}

The employed HIL facility is depicted in Fig. 4. The test bed is based on the slip control boost technology from ZF TRW Automotive (Koblenz, Germany). It is suitable for testing several operation modes, including base brake and ABS control. The experimental setup contains EHB, its control unit, and the host personal computer, which runs the IPG CarMaker $^{\circledR}$ vehicle model. This latter is interfaced to the EHB through the $\mathrm{dSPACE}^{\circledR}$ (Paderborn, Germany) electronic platform. The $\mathrm{dSPACE}^{\circledR}$ receives the demanded brake pressure signal from the numerical model and converts it into a signal

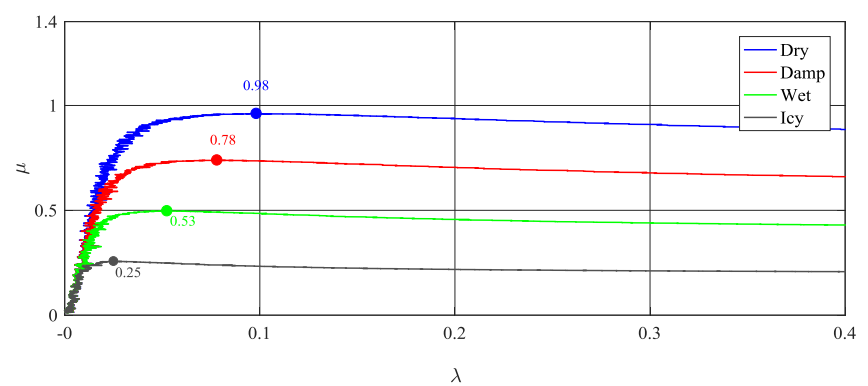

Fig. 3. Tires' slip-force curves with optimal wheel slip values for various road surfaces modeled with Pacejka's Magic Formula 6.1.
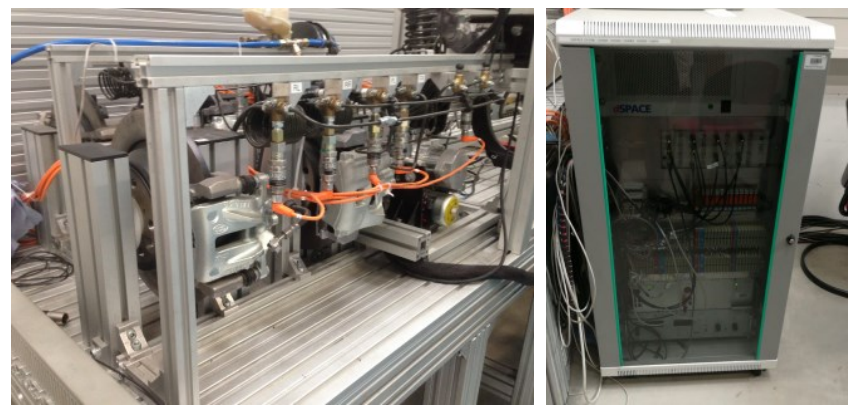

Fig. 4. EHB hardware-in-the-loop experimental setup. On the left, EHB system; on the right $\mathrm{dSPACE}^{\circledR}$ box.

for the EHB control unit. Afterwards, the measured brake line pressure from each caliper is fed back to the host computer and, thereafter, employed in the vehicle dynamics simulation software. The signals are sampled at $1 \mathrm{~ms}(1 \mathrm{kHz})$.

\section{FUZZY LOGIC CONTROLLER}

The main task of the designed $\mathrm{ABS}$ is to request an appropriate torque from the actuators in order to maintain the optimal slip for each wheel on varying road surfaces. The developed ABS relies on the CAN bus vehicle body acceleration $\left(a_{V x}\right)$ and wheel speed $\left(\omega_{W}\right)$ signals, which are available in modern cars (Fig. 2) [14]. Thereafter, applying (2) - (5), the controller's variables, $\lambda$ and $\mu^{*}$, are computed. Two separate FLCs are built respectively for the SRMs and the EHB testbed. The reader is referred to [3] for a better explanation of the control method and FLCs' design. In this paper, only a short description is provided.

The control method based on fuzzy set theory is designed to receive the information about the vehicle body deceleration rate at the beginning of a heavy braking maneuver. Its peak value is directly related to the road surface under the wheels. As the variable has vague and ill-defined characteristics, artificial decision making system, like fuzzy logic systems, evolutionary computation, computational swarm intelligence, etc., prove to be an effective tool for road classification, whereby a precise mathematical model might fail. The second input is the estimated wheel slip, which is necessary to understand if the torque must be increased or decreased to achieve the optimal value. Therefore, each FLC fuses two

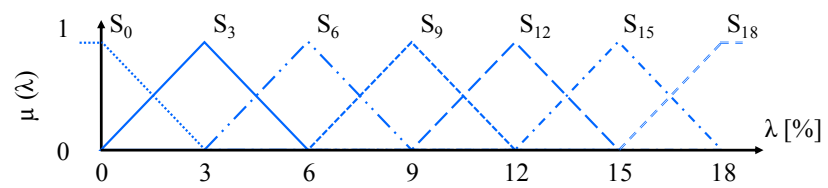

(a)

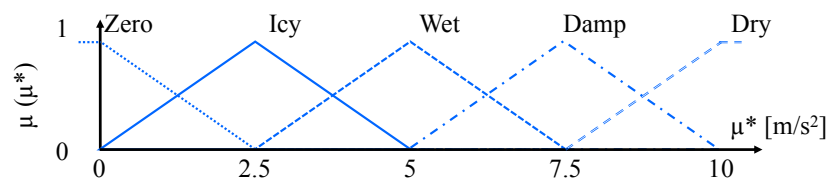

(b)

Fig. 5. FLC MFs for a single wheel: (a) wheel slip; (b) road surface. 
TABLE I. FLC RULE BASE FOR FRONT / REAR WHEELS IN REGENERATIVE BRAKING MODE

\begin{tabular}{|c|c|c|c|c|c|c|}
\hline \multicolumn{2}{|c|}{$\boldsymbol{T}_{\boldsymbol{R} B}[\mathbf{N m}]$} & \multicolumn{5}{|c|}{$\boldsymbol{\mu}^{*}\left[\mathrm{~m} / \mathbf{s}^{2}\right]$} \\
\cline { 3 - 7 } & Zero & Icy & Wet & Damp & Dry \\
\hline \multirow{4}{*}{$\boldsymbol{\lambda}$} & $\boldsymbol{S}_{\boldsymbol{0}}$ & 60 & 80 & 160 & $200 / 120$ & $200 / 140$ \\
\cline { 2 - 7 } & $\boldsymbol{S}_{\boldsymbol{3}}$ & 40 & 60 & 140 & $200 / 100$ & $200 / 120$ \\
\cline { 2 - 7 } & $\boldsymbol{S}_{\boldsymbol{6}}$ & 20 & 40 & 120 & $200 / 80$ & $200 / 100$ \\
\cline { 2 - 7 }$[\%]$ & $\boldsymbol{S}_{\boldsymbol{9}}$ & 0 & 20 & 100 & $180 / 40$ & $200 / 80$ \\
\cline { 2 - 7 } & $\boldsymbol{S}_{12}$ & 0 & 0 & 60 & $160 / 20$ & $200 / 40$ \\
\cline { 2 - 7 } & $\boldsymbol{S}_{15}$ & 0 & 0 & 20 & $140 / 0$ & $180 / 20$ \\
\cline { 2 - 7 } & $\boldsymbol{S}_{18}$ & 0 & 0 & 0 & $120 / 0$ & $160 / 0$ \\
\hline
\end{tabular}

inputs (i.e. wheel slip and road surface) into one output (i.e. requested braking torque for the SRM or EHB actuator). Therefore, the controller has a multi input, single output form. It does not involve a reference set point. Due to plant complexity and high nonlinearity, the studied FLC is designed referring to expert's knowledge.

The FLC's first interface is fuzzification, which converts "crisp" inputs into fuzzy sets. In Fig. 5, the input MFs are depicted. For the sake of simplicity, all the MFs have triangular shapes: $\lambda$ has five, $\mu^{*}-$ seven. They are symmetrically dispersed over the whole universe of discourse and are overlapped to ensure equal input sensitivity. The universe of discourse of the wheel slip is limited inside the range $[0,18]$, while the road estimator in the range $[0,10]$.

Using pre-defined rule base, the FLC maps the inputs with the consequent linguistic value outputs through the inference mechanism. Sugeno's inference method is applied. The rule base for the front and rear wheels in regenerative braking mode is delivered in Table I. It equivalently represents an expert driver's knowledge of the plant control strategy. The output torque has eleven output values from 0 (i.e. no torque requested) to 200 (i.e. maximum available torque for the SRM in $\mathrm{Nm}$ ). The values are equally separated with a step of $20 \mathrm{Nm}$. The $T_{F B}$ is restricted between 0 (i.e. no pressure is required to the EHB) and 150, which is maximum EHB's pressure in bar. The values are equally distributed with a step of $10 \mathrm{bar}$. The rule base for front and rear wheels is introduced in Table II.

The tabular rule base is expressed in modus ponens syntax (If premise Then consequence). The rule based is obtained with trial and error method with the aim of reaching a wheel slip as close as possible to the optimal value for each wheel

TABLE II. FLC RULE BASE FOR FRONT / REAR WHEELS IN FRICTION BRAKING MODE

\begin{tabular}{|c|c|c|c|c|c|c|}
\hline \multirow{2}{*}{$\boldsymbol{T}_{\boldsymbol{F B}}[\mathbf{N m}]$} & \multicolumn{5}{|c|}{$\boldsymbol{\mu}^{*}\left[\mathbf{m} / \mathbf{s}^{\mathbf{2}}\right]$} \\
\cline { 2 - 7 } & Zero & Icy & Wet & Damp & Dry \\
\hline \multirow{4}{*}{$\boldsymbol{\lambda}$} & $\boldsymbol{S}_{\boldsymbol{0}}$ & 20 & 30 & 60 & $90 / 70$ & $150 / 90$ \\
\cline { 2 - 7 } & $\boldsymbol{S}_{\boldsymbol{3}}$ & 10 & 20 & 50 & $80 / 50$ & $130 / 80$ \\
\cline { 2 - 7 } & $\boldsymbol{S}_{\boldsymbol{6}}$ & 0 & 10 & 30 & $70 / 30$ & $110 / 70$ \\
\cline { 2 - 7 }$[\mathbf{\%}]$ & $\boldsymbol{S}_{\boldsymbol{9}}$ & 0 & 0 & 10 & $50 / 10$ & $90 / 50$ \\
\cline { 2 - 7 } & $\boldsymbol{S}_{\boldsymbol{1 2}}$ & 0 & 0 & 0 & $30 / 0$ & $60 / 30$ \\
\cline { 2 - 7 } & $\boldsymbol{S}_{15}$ & 0 & 0 & 0 & $10 / 0$ & $30 / 10$ \\
\cline { 2 - 7 } & $\boldsymbol{S}_{\boldsymbol{1 8}}$ & 0 & 0 & 0 & $0 / 0$ & $0 / 0$ \\
\hline
\end{tabular}

and for different road adhesion coefficients. An example of input-output linguistic mapping is as follows: If wheel "slip equals to 6" and road surface is "Icy", Then request from the $\mathrm{SRM}$ a regenerative braking torque equal to " 40 " $\mathrm{Nm}$ (Table I). Finally, the defuzzification step converts the linguistic output set into numerical values. The center of gravity approach is used.

\section{STABILITY AND PERFORMANCE ANALYSIS}

The vehicle is a highly nonlinear system with time varying parameters. During braking occurrence, owing to the relatively low wheels' moment of inertia, the wheel angular speed features very fast dynamics. Rapid brake actuations occurring during ABS intervention stress these dynamics even more. In this regard, an exceedance of the optimal tire slip for a specific road might lead the system to an unstable behavior, which appears as cyclic wheel lock and release.

In this section, the authors address the stability problem under a graphic perspective showing that the choice of the equilibrium point strongly affects the stability of the system. The system is unstable if the control action cannot keep the operating point on the TPP diagram close to the equilibrium. On the other hand, if the curve converges to the equilibrium point, the system is stable.

The time differentiation of (3) leads to the next statement:

$$
\dot{\lambda} v_{V x}+\lambda \dot{v}_{V x}=\dot{v}_{V x}-\dot{\omega}_{W} r_{W}
$$

By placing (1) into (6), the following longitudinal wheel slip dynamics equation is derived:

$$
\dot{\lambda}=\left(\frac{T_{B}-F_{X}(\lambda) \cdot r_{W}}{J_{W}}\right) \cdot \frac{r_{W}}{v_{V x}}+\frac{\dot{v}_{V x}}{v_{V x}} \cdot(1-\lambda) .
$$

The equilibrium points for (7) are characterized by $\dot{\lambda}=0$, where the following condition holds true:

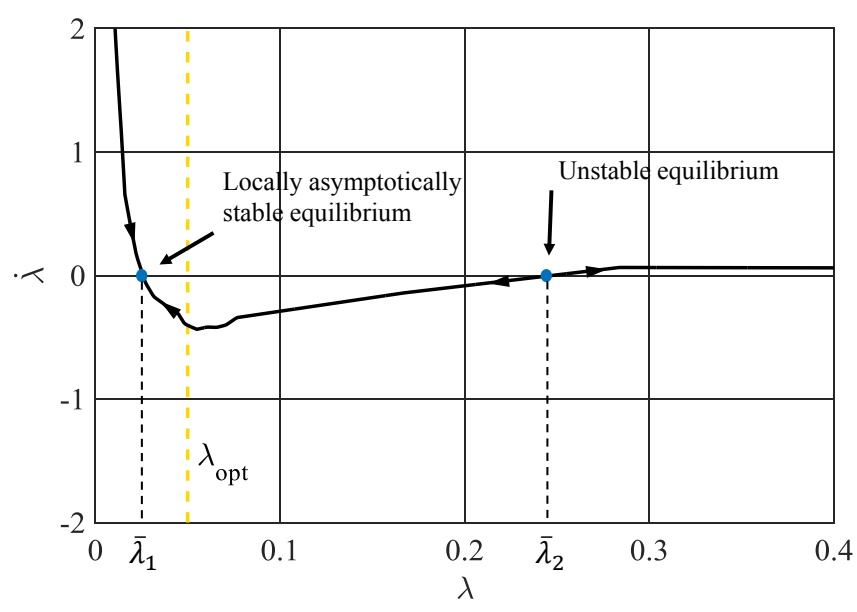

Fig. 6. Phase-plane diagram of slip ratio vs slip ratio derivative for constant braking torque on wet road derived from (7). The solutions to the (8), namely $\bar{\lambda}_{1}$ and $\bar{\lambda}_{2}$, are reported as blue dots [9]. 


$$
\bar{T}_{B}=-\dot{v}_{V x} \cdot(1-\bar{\lambda}) \cdot \frac{J_{w}}{r_{w}}+F_{X}(\bar{\lambda}) \cdot r_{w} .
$$

Equation (8) has zero, one or two solutions, depending on the applied torque value. In Fig. 6, (7) is represented for a constant applied torque value on wet road, intercepting two equilibria in accordance with (8). As can be seen from Fig. 6, $\bar{\lambda}_{1}$ is a locally stable equilibrium, while $\bar{\lambda}_{2}$ is unstable. Indeed, small variations in the tire slip from the equilibrium $\bar{\lambda}_{2}$ might induce an unstable dynamics that, in turn, leads the system to wheel lockage. Such a condition should be avoided to maximize the brake effectiveness.

This reasoning can be extended to any other set of equilibria showing that the system has stable behavior, if the equilibrium point associated with a slip value falls on the left side of the slip-force curve peak. To the limit, when the equilibrium corresponds to the optimal slip (peak of the slipforce curve), which provides the maximum braking effectiveness, the system becomes unstable as soon as the slip exceeds the optimum rate. These results led to a conservative choice of the reference slip value set in the FLC, which always falls to the left side of the slip-force curve peak.

\section{RESUlts}

The HIL simulation results conducted on a low $-\mu$ surface (i.e. $\mu=0.5$, wet asphalt) are reported in Fig. 7 for FB and in Fig. 8 for RB. The simulations refer to emergency braking on a straight road. In Fig. 7 (b) and 8 (b), the slip curves are introduced. It is noticeable that for each wheel the slip value in case of RB (Fig. 8 (b)) is closer to the optimal one, compared to the case with FB (Fig. 7 (b)).

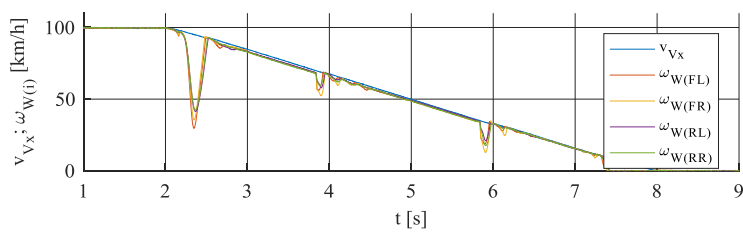

(a)

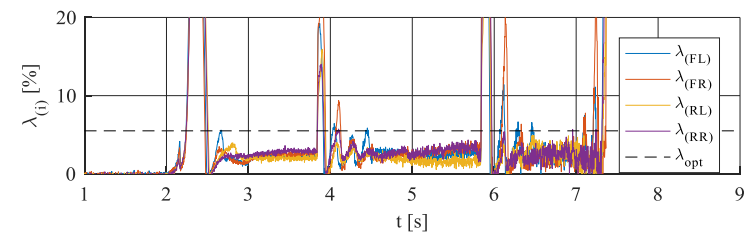

(b)

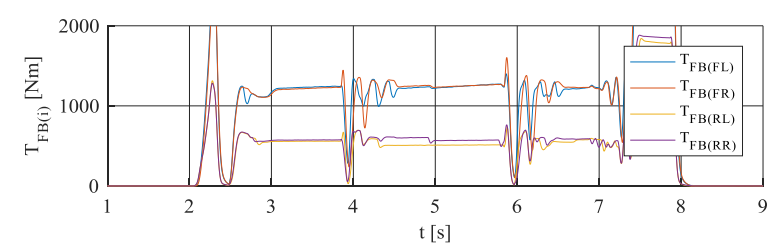

(c)

Fig.7. Experimental results from braking on low $-\mu$ road surface (i.e. $\mu=0.5$, wet asphalt) for FB: (a) vehicle and wheels speeds; (b) wheels longitudinal slips; (c) FB torques.
In Fig. 9, the stability analysis based on TPP is scoped. For the sake of clarity, the analysis is limited to the front-left wheel. Three different braking configurations are depicted on the phase-plane diagram: i) disabled ABS, ii) ABS with full utilization of conventional brake; iii) ABS with RB. The arrows show the magnitude and direction of the wheel slip variation. In addition, the optimal wheel slip (Fig. 3) for the wet road surface is emphasized by the vertical yellow dotted line.

When the ABS is disabled (Fig. 9, red trajectory), the system has an unstable behavior which corresponds to wheel lock. As a result, the vehicle steerability is no longer ensured. The second case is ABS control with pure FB utilization (Fig. 9, black trajectory). Although the system reaches its stable equilibrium point, the FLC controlling the EHB takes significant time to reach the equilibrium. The resulting slow dynamics is mainly attributable to the actuation delay of the EHB test bed. When the equilibrium point is reached, the derivative of the wheel slip ratio is close to zero. At last, the third case corresponds to ABS control by regenerative braking (Fig. 9, blue trajectory). Thanks to their fast dynamics, the electric actuators allow for a faster convergence of the system to the equilibrium.

The developed FLCs allows for stability independently of the employed actuator (i.e. EHB or SRM); indeed, the TPP converges to the equilibrium point. The FLCs operate within a safe slip margin, before the unstable slip-force region is reached. The radius of the resulting TPP is an effective indication of the controller performance. The slow dynamics of the EHB, compared to the SRM, impacts the vehicle safety as the equilibrium lies far from the optimal wheel slip. The electric actuators outperform the friction brakes allowing for a faster convergence to the equilibrium. Furthermore, the electric

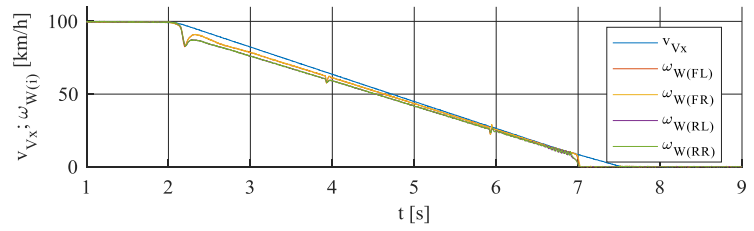

(a)

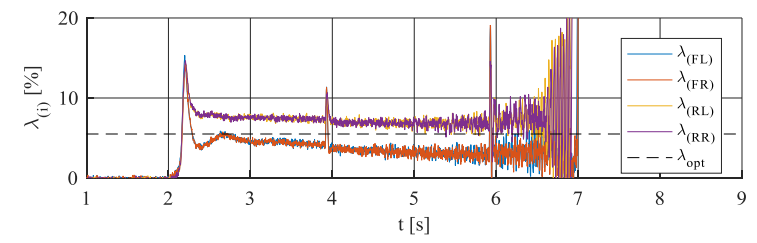

(b)

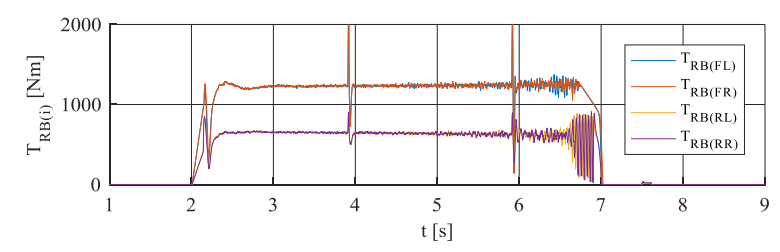

(c)

Fig.8. Experimental results from braking on low $-\mu$ road surface (i.e. $\mu=0.5$, wet asphalt) for RB: (a) vehicle and wheels speeds; (b) wheels longitudinal slips; (c) RB torques. 
actuators ensure also better tracking of the optimal tire slip thanks to their faster dynamics.

The TPP method allows for visual representation of controller's stability and performance, nevertheless, it cannot prove asymptotic stability of the controller. Although it might be argued that it is possible to define a stability analysis to design the rule base of FLC, the classical stability analysis introduced by Lyapunov cannot be applied to an FLC, as this latter is a task-oriented control, whilst conventional control is characterized as set point-oriented control [10]. Therefore, the TPP may be successfully applied to analyse control stability and performance of complex nonlinear systems. Moreover, the TPP method constitutes a valid alternative to the time-domain observation of an ABS slip tracking performance.

\section{CONCLUSION}

The present study is aimed at analyzing the TPP method to visually assess the stability properties and convergence performance of an FLC for an ABS. Herein, the investigated controller is designed for vehicle ABS so that the closed loop wheel slip dynamics is stable around the equilibrium. The results have shown that the TPP method reveals very effective at providing a visual representation of the controller's stability and performance analysis for highly nonlinear systems, such as ABS control. Consequently, the TTP method is particularly suitable for non-linear systems whereby analytical solutions might not exist.

The FLC is tested under HIL simulations conducted at the Technische Universität Ilmenau. The stability and performance of the ABS are examined against several control configurations: i) disabled ABS; ii) ABS with full utilization of conventional brake; iii) ABS with regenerative braking. The analysis presented in this work justify that: (i) the convergence of the developed FLC for ABS is graphically assessed through the TPP; (ii) the electric motors outperform the electrohydraulic brakes by allowing for a faster convergence; (iii) the electric motors ensure better tracking of the optimal tire slip

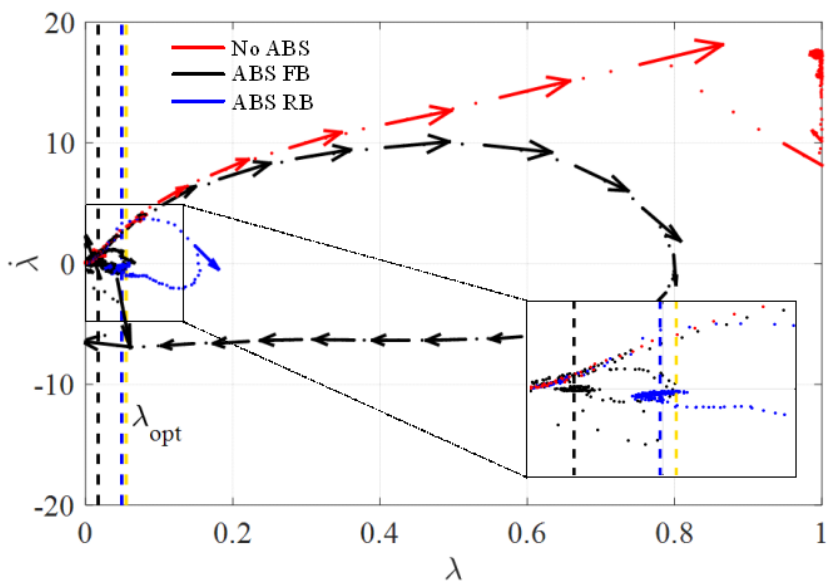

Fig. 9. Phase-plane diagram of slip ratio vs slip ratio derivative for the three considered configuration: "No ABS" - deactivated ABS leads to wheel lock; "ABS FB" - friction braking ABS (Fig. 7); "ABS RB" regenerative braking ABS (Fig. 8). In the small box at the bottom left, the convergence values for each case are reported (in blue ABS in pure regeneration and in black $\mathrm{ABS}$ with conventional friction brakes). thanks to their faster dynamics.

In the future, the control method's functionality together with the stability and performance analysis will be conducted for more complex maneuvers, like split- $\mu$ road surface (i.e. half of the road surface has different adhesion coefficient than the other one), braking while turning, transient road conditions, etc. Furthermore, it is planned to carry out similar study on a real SUV with four on-board motors powertrain.

\section{ACKNOWLEDGEMENT}

This project has received funding from the European Union's Horizon 2020 research and innovation program under grant agreement No. 675999.

\section{REFERENCES}

[1] W. Post, "Car Braking Systems," in Brakes, Brake Control and Driver Assistance Systems: Function, Regulation and Components, K. Reif, Ed., Wiesbaden, Germany: Springer Vieweg, 2014, pp. 28-39.

[2] D. Savitski, V. Ivanov, B. Shyrokau, T. Pütz, J. De Smet, and J. Theunissen, "Experimental investigations on continuous regenerative anti-lock braking system of full electric vehicle," Int. J. Automotive Technology, vol. 17, pp. 327-338, April 2016.

[3] A. Aksjonov, V. Vodovozov, K. Augsburg, and E. Petlenkov, "Design of regenerative anti-lock braking system controller for 4-in-wheelmotor drive electric vehicle with road surface estimation," Int. J. Automotive Technology, vol. 19, pp. 727-742, August 2018.

[4] D. Paul, E. Velenis, D. Cao, and T. Dobo, "Otpimal $\mu$-estimation based regenerative braking strategy for an AWD HEV," IEEE Trans. on Transp. Electrif., vol. 3, pp. 249-258, March 2017.

[5] G. F. Mauer, "A fuzzy logic controller for an ABS braking system," IEEE Trans. on Fuzzy Syst., vol. 3, pp. 381-388, November 1995.

[6] J. A. Cabrera, A. Ortiz, J. J. Castillo, and A. Simón, “A fuzzy logic control for antilock braking system integrated in the IMMa tire test bench," IEEE Trans. on Veh. Tech., vol. 54, pp. 1937-1949, November 2005 .

[7] J. Guo, X. Jian, and G. Lin, "Performance evaluation of an anti-lock braking system for electric vehicle with fuzzy sliding mode controller," Enegies (MDPI), vol. 7, pp. 6459-6476, August 2014.

[8] J. J. Castillo, J. A. Cabrera, A. J. Guerra, and A. Simón, "A novel electrohydraulic brake system with tire-road friction estimation and continuous brake pressure control," IEEE Trans. on Indust. Electr., vol. 63, pp. 1863-1875, March 2016.

[9] S. M. Savaresi and M. Tanelli, Active Braking Control Systems Design for Vehicles, London, UK: Springer-Verlag, 2010.

[10] O. Castillo, Type-2 Fuzzy Logic in Intelligent Control Applications, Berlin: Springer-Verlag Berlin Heidelberg, 2012, pp. 49-61.

[11] K.M. Passino and S. Yurkovich, Fuzzy Control, Menlo Park, California, USA: Addison-Wesley, 1998, pp. 188-225.

[12] M. Doumiati, A. Charara. A. Victorino, and D. Lechner, Vehicle Dynamics Estimation using Kalman Filtering: Experimental Validation, London: ISTE Ltd and John Wiley \& Sons, Inc., 2012.

[13] U. Kiencke and L. Nielsen, Automotive Control Systems: For Engine, Driveline, and Vehicle, 2nd ed., Berlin: Springer-Verlag Berlin Heidelberg, pp. 301-350, 351-408, 409-424, 2005.

[14] E. Zabler, "Sensors for Brake Control," in Brakes, Brake Control and Driver Assistance Systems: Function, Regulation and Components, K. Reif, Ed., Wiesbaden, Germany: Springer Vieweg, 2014, pp. 142-153.

[15] H. B. Pacejka, Tyre and Vehicle Dynamics, 3rd ed., Oxford: Butterworth-Heinemann, 2012.

[16] V. Ricciardi, D. Savitski, K. Augsburg, and V. Ivanov, "Estimation of brake friction coefficient for blending function of base braking control," SAE Int. J. Passeng. Cars - Mech. Syst., vol. 10, pp. 774-785, 2017. 